\title{
The Correlation Between Platelet-to-Lymphocyte Ratio and Neutrophil-to-Lymphocyte Ratio with Hepatic Echinococcosis
}

\author{
Weijian $E^{\prime}$ \\ Zhixin Wang ${ }^{2}$ \\ Mingquan Pang ${ }^{2,3}$ \\ Yongliang Lu' \\ Haining $\operatorname{Fan}^{2,3}$ \\ 'Centre of Medical Laboratory \\ Technology \& Laboratory Diagnostics, \\ Affiliated Hospital of Qinghai University, \\ Xining, Qinghai, 8I000I, People's \\ Republic of China; ${ }^{2}$ Department of \\ Hepatopancreatobiliary Surgery, \\ Affiliated Hospital of Qinghai University, \\ Xining, Qinghai, 8I000I, People's \\ Republic of China; ${ }^{3}$ Qinghai Provincial \\ Research Key Laboratory for Hydatid, \\ Xining, Qinghai, 81000I, People's \\ Republic of China
}

Objective: To explore the correlation between neutrophil-to-lymphocyte ratio (NLR) and platelet-to-lymphocyte ratio (PLR) with hepatic hydatid diseases.

Methods: The clinical data of 420 patients with hydatid disease who were treated in our hospital from October 2015 to October 2018 were collected from the database of our hospital. According to the postoperative pathological diagnosis, 200 patients were assigned into the alveolar echinococcosis (AE) group, and 220 patients were assigned into the cystic echinococcosis (CE) group. A total of 160 healthy examinees were enrolled as the control group. The main observation indexes included preoperative PLR and NLR. Pearson's correlation was used to analyze the correlation between PLR and NLR with clinical indicators in HE patients, and the receiver operator characteristic (ROC) curve was used to evaluate the clinical values of PLR and NLR in diagnosing different types of hydatid diseases.

Results: The results revealed that the expressions of PLR and NLR were significantly higher in the AE group than in the $\mathrm{CE}$ and control groups $(\mathrm{P}<0.05)$. The results of multiple linear regression analysis revealed that PLR was positively correlated with aspartate aminotransferase, alanine aminotransferase, total bilirubin, direct bilirubin, alkaline phosphatase and $\gamma$ glutamyltranspeptidase $(\mathrm{P}<0.05)$. NLR was correlated with albumin content $(\mathrm{P}<0.05)$. ROC curve analysis results revealed that the area under the curve (AUC) of PLR and NLR for AE was 0.800 and 0.700 respectively, and the AUC for CE was 0.78 and 0.75 respectively.

Conclusion: The NLR and PLR of AE patients were higher than those of CE patients, and the high inflammation of $\mathrm{CE}$ patients may be correlated to the reproductive mode of Echinococcus multilocularis. PLR and NLR have certain diagnostic values for disease classification, but PLR has higher specificity when compared with NLR.

Keywords: platelet-to-lymphocyte ratio, PLR, neutrophil-to-lymphocyte ratio, NLR, alveolar echinococcosis, cystic echinococcosis, inflammation

\section{Introduction}

Hepatic echinococcosis (HE), is a zoonotic parasitic disease. The parasites are spread between canines and domestic animals, especially sheep, and human beings are infected as occasional intermediate hosts. This infection is widespread around the world, especially the rural animal husbandry areas in the Mediterranean, Eastern Europe, North Africa and East Africa, South America, Central Asia, China and Australia, and China's Xinjiang, Ningxia, Qinghai and Tibet and other northwest provinces are high incidence areas. ${ }^{1}$ According to the types of parasites infected by the patients, HE is mainly divided into two types: the hepatic cystic echinococcosis 
(HCE) caused by the young Echinococcus granulosus, and the hepatic alveolar echinococcosis (HAE) caused by the young Echinococcus multilocularis. HCE mainly occurs in the liver, followed by the lungs. It is a chronic, clinically complex and easily neglected disease. ${ }^{2}$ Its clinical manifestations range from asymptomatic disease to serious and even life-threatening diseases, and most patients still have one or more symptoms for several years or even decades and maybe diagnosed accidentally. HAE is more serious than HCE and is characterized by slow and occult onset, exhibits infiltrating growth, invades adjacent organs and migrates to distant organs. ${ }^{3}$ The prognosis of HAE is similar to that of a liver tumor. The mortality rate of untreated patients is more than $90 \%$ within $10-15$ years after diagnosis. $^{4}$

Hepatic hydatid disease is a parasitic disease where Echinococcus invades the human body. It leads to a corresponding immune response as an exogenous antigen. However, the survival of Echinococcus in the human body is related to its specific immune escape system. It has been reported that $\mathrm{CD} 4+$ helper $\mathrm{T}$ cells play an important role in chronic Echinococcosis, Th1/Th2 shift and Th17/ Treg cell expression imbalance occurs in peripheral blood after hydatid infection, which is related to the tendency of chronic pathogenesis of hydatid infection. ${ }^{5,6}$ Another study revealed that platelets played a dual role in the regulation of tissue inflammation: during the beginning of inflammation, many soluble substances produced by platelets induced the proliferation, differentiation and migration of Th1 and Th17 cells; while during the vanishing of inflammation, Th1 and Th17 differentiation were inhibited by the formation of platelet-T cell aggregates. And the ratio of Th2 to Treg cells increased when platelets were abundant. ${ }^{7}$ Inflammation is an important characteristic of parasite infection, neutrophils, lymphocytes, monocytes, platelets and other hematological inflammatory parameters can reflect the immune status of the body and are involved in the chronic inflammatory response of Echinococcosis, which may be related to the immune escape mechanism of hepatic hydatid.

The neutrophil-to-lymphocyte ratio (NLR) and platelet-to-lymphocyte ratio (PLR), which can reflect inflammation and the host immune response, are considered as new markers of the systemic inflammatory index in chronic diseases. They are related to the incidence and mortality of many chronic diseases such as diabetes, hypertension and cardiovascular disease, which are also new prognostic indicators of various malignant tumors. ${ }^{8}$
NLR has been introduced as a marker in various conditions in literature. These include type 2 diabetes mellitus, ${ }^{9}$ thyroiditis, ${ }^{10}$ ulcerative colitis, ${ }^{11}$ hepatosteatosis, ${ }^{12}$ and malignancy. ${ }^{13}$ Moreover, NLR has been correlated with glycated hemoglobin (HbAlc) in diabetic subjects. ${ }^{14}$ NLR and PLR have been accepted as markers of subclinical inflammation and are used in combination with other inflammatory markers to determine the degree of inflammation in many diseases. However, the pattern and the possible role of PLR and NLR in two types of Echinococcosis are poorly understood.

Therefore, this study was designed to explore the correlation between PLR and NLR with hepatic hydatid diseases.

\section{Data and Methods}

\section{Subjects}

This study retrospectively analyzed the clinical data of HE patients who were admitted to the Affiliated Hospital of Qinghai University from October 2015 to October 2018, including the general information of the patients, their current medical history, laboratory examination data and medical records. According to the postoperative pathological diagnosis, these 200 patients were assigned to the alveolar echinococcosis (AE) group, and 220 patients were assigned to the cystic echinococcosis (CE) group. Some healthy physical examinees were enrolled as the control group. The Ethics Committee of our hospital approved this study, and all patients provided signed informed consent.

\section{Inclusion and Exclusion Criteria}

Inclusion criteria: (1) Patients with diagnosed HE; (2) the age of the patients was $>18$ years old; and (3) patients with complete clinical data. Exclusion criteria: (1) patients complicated with viral infection, bacterial infection, mycoplasma or chlamydia infection, infectious diseases such as high fever, autoimmune diseases, malignant tumors, cardiovascular and cerebrovascular diseases, and renal insufficiency; (2) patients with severe obstructive jaundice; (3) Those who took anti-inflammatory drugs, immunosuppressants and anti-parasitic drugs within 3 months before diagnosis; (4) patients with other parasitic diseases.

\section{Main Observation Indices}

The main observation indexes of this study were the PLR and NLR in peripheral blood in the three groups before the operation. 


\section{Statistical Analysis}

Data were analyzed using statistical software SPSS 20.0. Measurement data were expressed as mean \pm standard deviation, and count data were expressed as a percentage (\%). The normality of variables was tested using the W-test. The homogeneity of the variances was tested using the F-test. Multi-group comparison of the measurement data was conducted using univariate analysis of variance, and post-hoc test was conducted using the least significant difference (LSD). Non-normally distributed mean or normally distributed mean with the heterogeneity of multiple samples were evaluated using a non-parametric test. Count data were evaluated using the Chi-square test. Pearson's correlation was used to analyze the correlation between PLR and NLR with clinical indicators in HE patients, and the receiver operator characteristic (ROC) curve was used to evaluate the clinical values of PLR and NLR in diagnosing different types of hydatid diseases. $\mathrm{P}<0.05$ was considered statistically significant.

\section{Results}

\section{Comparison of Basic Clinical Data Among the Three Groups}

In Table 1, the data of $420 \mathrm{HE}$ patients (including $200 \mathrm{AE}$ patients and $220 \mathrm{CE}$ patients) and 160 healthy participants were retrospectively analyzed. There was no significant difference in age and gender among the three groups. In the AE group, the total number of white blood cells was 7.15 $\pm 2.60 \times 10^{9}$, the neutrophil count was $4.40 \pm 2.19 \times 10^{9}$, and the platelet count was $218.66 \pm 61.09 \times 10^{9}$, which was significantly higher compared with those in the control group $(\mathrm{P}<0.05)$. In the $\mathrm{CE}$ group, the total number of white blood cells was $6.52 \pm 2.34 \times 10^{9}$, the neutrophil count was 3.87 $\pm 2.01 \times 10^{9}$, and the platelet count was $235.18 \pm 87.21 \times 10^{9}$, which was significantly higher compared with those in the control group $(\mathrm{P}<0.05)$. In the $\mathrm{AE}$ group, the total number of red blood cells $(\mathrm{RBC})$ was $4.51 \pm 0.82 \times 10^{12}$, hemoglobin was $126.51 \pm 26.58 \mathrm{~g} / \mathrm{L}$, which was significantly higher compared with those in the control group $(\mathrm{P}<0.05)$. In the $\mathrm{CE}$ group, the total number of RBC was $4.90 \pm 0.70 \times 10^{12}$, hemoglobin was $142.73 \pm 25.13 \mathrm{~g} / \mathrm{L}$, which was significantly higher compared with those in the control group $(\mathrm{P}<0.05)$. In the $\mathrm{AE}$ group, the total number of lymphocytes was 1.73 $\pm 0.68 \times 10^{9}$, and in the $\mathrm{CE}$ group the total number of lymphocytes was $1.88 \pm 0.71 \times 10^{9}$, compared with the control group, the difference was not statistically significant $(\mathrm{P}=0.133)$.

Compared with the control group, the expression levels of aspartate aminotransferase (AST, $38.68 \pm 35.58 \mathrm{vs} 23.24$ \pm 6.77 ), alanine aminotransferase (ALT, 38.15 $\pm 39.10 \mathrm{vs}$

Table I Comparison of Baseline Data the Three Groups $(\bar{x} \pm s)$

\begin{tabular}{|c|c|c|c|c|}
\hline Index & Alveolar Echinococcosis $(n=200)$ & Cystic Echinococcosis $(n=220)$ & Healthy Control Group $(n=160)$ & $\mathbf{P}$ \\
\hline Gender (M/F) & $67 / 133$ & $90 / 130$ & $65 / 95$ & 0.473 \\
\hline Age (years) & $37.08 \pm 13.85$ & $38.72 \pm 16.69$ & $39.27 \pm 15.77$ & 0.504 \\
\hline ALT (U/L) & $41.38 \pm 42.74$ & $35.34 \pm 35.30$ & $22.49 \pm 11.18$ & 0.0001 \\
\hline AST (U/L) & $44.82 \pm 42.32$ & $33.12 \pm 27.03$ & $23.24 \pm 6.77$ & 0.0001 \\
\hline TBIL (U/L) & $41.22 \pm 77.79$ & $16.8 \mid \pm 27.66$ & $15.27 \pm 6.36$ & 0.0001 \\
\hline DBIL (U/L) & $27.46 \pm 57.69$ & $7.98 \pm 21.56$ & $2.96 \pm 1.83$ & 0.0001 \\
\hline IBIL (U/L) & $14.97 \pm 29.88$ & $9.03 \pm 8.04$ & $12.36 \pm 5.55$ & 0.001 \\
\hline TP (U/L) & $72.22 \pm 10.29$ & $68.83 \pm 7.57$ & $77.96 \pm 47.80$ & 0.0001 \\
\hline ALB (U/L) & $35.47 \pm 5.36$ & $38.96 \pm 5.01$ & $45.94 \pm 3.93$ & 0.0001 \\
\hline ALP (U/L) & $287.72 \pm 316.16$ & $134.92 \pm 108.02$ & $94.89 \pm 56.56$ & 0.0001 \\
\hline GGT (U/L) & $150.73 \pm 184.73$ & $67.48 \pm 79.50$ & $20.04 \pm 15.07$ & 0.0001 \\
\hline WBC $\left(10^{9} / \mathrm{L}\right)$ & $7.15 \pm 2.60$ & $6.52 \pm 2.34$ & $6.14 \pm 1.52$ & 0.0001 \\
\hline NEU $\left(10^{9} / L\right)$ & $4.40 \pm 2.19$ & $3.87 \pm 2.01$ & $3.55 \pm 1.11$ & 0.0001 \\
\hline $\operatorname{LYM}\left(10^{9} / \mathrm{L}\right)$ & $1.73 \pm 0.68$ & $|.88 \pm 0.7|$ & $2.15 \pm 0.65$ & 0.030 \\
\hline $\operatorname{RBC}\left(10^{9} / \mathrm{L}\right)$ & $4.5 I \pm 0.82$ & $4.90 \pm 0.70$ & $5.14 \pm 0.50$ & 0.0001 \\
\hline HGB $(g / L)$ & $|26.5| \pm 26.58$ & $142.73 \pm 25.13$ & $157.43 \pm 16.83$ & 0.0001 \\
\hline PLT $\left(10^{9} / \mathrm{L}\right)$ & $218.66 \pm 61.09$ & $235.18 \pm 87.21$ & $255.16 \pm 94.85$ & 0.0001 \\
\hline PLR & $163.96 \pm 72.3$ & $|35.04 \pm 65.3|$ & $106.15 \pm 34.78$ & 0.0001 \\
\hline NLR & $2.94 \pm 2.17$ & $2.56 \pm 2.59$ & $1.78 \pm 0.76$ & 0.0001 \\
\hline
\end{tabular}

Abbreviations: ALT, alanine transaminase; AST, glutamic oxalacetic transaminase; TBIL, total bilirubin; DBIL, direct bilirubin; IBIL, indirect bilirubin; TP, total protein; ALB, albumin; ALP, alkaline phosphatase; GGT, glutamyl transpeptidase; RBC, red blood cell count; HGB, red blood cell count; WBC, white blood cell count; NEU, central granulocyte count; LYM, lymphocyte count; PLT, blood platelet count. 
$22.49 \pm 11.18$ ), total bilirubin (TIBL, $28.40 \pm 58.46$ vs 15.27 \pm 6.36 ), direct bilirubin (DBIL, $17.23 \pm 43.79$ vs $2.96 \pm$ 1.83), alkaline phosphatase $(207.47 \pm 242.95$ vs $94.89 \pm$ 56.56), and $\gamma$-glutamyltranspeptidase (GGT, $107.01 \pm$ 145.30 vs $20.04 \pm 15.07$ ) were significantly higher in the HE group (that is the Echinococcosis group, including the $\mathrm{CE}$ and $\mathrm{AE}$ groups), and all the differences were statistically significant $(\mathrm{P}<0.05)$. Compared with the control group, the expression levels of indirect bilirubin (IBIL, $11.84 \pm 21.58$ vs $12.36 \pm 5.55$ ) and albumin (ALB, 37.30 \pm 5.46 vs $45.94 \pm 3.93$ ) were significantly lower in the HE group, and all the differences were statistically significant $(\mathrm{P}<0.000)$. However, the difference in the expression level of total protein between the control group and the HE group was not statistically significant (Table 1).

\section{Comparison of PLR and NLR Among Three Groups}

The average values of PLR and NLR were 106.15 \pm 34.78 and $1.78 \pm 0.76$, respectively, in the control group, $163.96 \pm 72.30$ and $2.94 \pm 2.17$, respectively in the $\mathrm{AE}$ group, and $135.04 \pm 65.31$ and $2.56 \pm 2.59$, respectively, in the CE group, the differences among the three groups were statistically significant $(\mathrm{P}<0.05)$. PLR and NLR were significantly higher in the $\mathrm{AE}$ group compared to those in the other two groups $(\mathrm{P}<0.05$, Figure 1$)$.

\section{Correlation Analysis of NLR and PLR with Clinical Indicators}

PLR and NLR were positively correlated with ALT, AST, IBIL and GGT and negatively correlated with ALB in peripheral blood, while NLR was positively correlated with age.

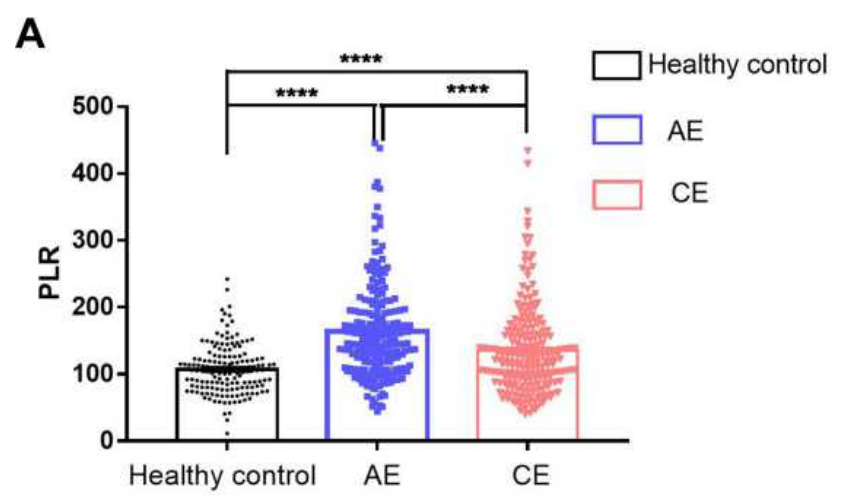

\section{ROC Analysis}

ROC curve analysis results revealed that the area under the curve (AUC) of PLR and NLR for AE was 0.800 and 0.700 respectively, and the AUC for $\mathrm{CE}$ was 0.78 and 0.75 , respectively.

\section{Discussion}

This study was designed to explore the correlation between PLR and NLR with hepatic hydatid diseases. The results of this study revealed that the expression levels of PLR and NLR were significantly higher in the AE group than in the $\mathrm{CE}$ and the control group. The results of multiple linear regression analysis also revealed that: PLR was positively correlated with AST, ALT, TIBL, DBIL, alkaline phosphatase and GGT, and NLR was correlated with ALB level. ROC curve analysis results revealed that the AUC of PLR and NLR for AE was 0.800 and 0.700 , respectively, and the AUC for CE was 0.78 and 0.75 , respectively.

As systemic inflammatory indexes related to the immune system, PLR and NLR can be directly obtained from the whole blood cell count, which is convenient and cheap. ${ }^{9,10}$ Many studies confirmed that one or two of PLR and NLR were closely related to the progress and prognosis of cardiovascular diseases and virus-related liver cancer. ${ }^{11,12}$ However, the related expression in HE has not been reported.

In this study, many patients who were pathologically diagnosed with hepatic $\mathrm{CE}$ and $\mathrm{AE}$ were retrospectively analyzed. The result revealed that the expressions of PLR and NLR were significantly higher in patients with hydatid disease. However, in the AE group, the lymphocyte count was significantly lower than in the other two groups, but the expression levels of platelet, neutrophil and PLR were significantly higher than those in the other two groups. Compared with the control group, the platelet and

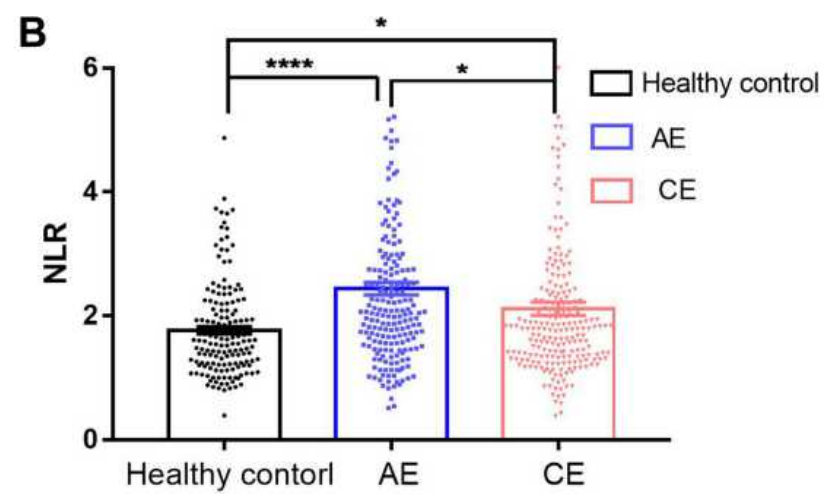

Figure I Comparison of PLR (A) and NLR (B) among three groups. The data are presented as mean \pm SEM. $* \mathrm{P}<0.05 ; * * * * \mathrm{P}<0.000 \mathrm{I}$ versus health control group. 
neutrophil counts were slightly higher, and the lymphocyte count was slightly lower in the CE group. The differences between the two groups were not statistically significant, but the difference in the increase in PLR and NLR between the two groups was statistically significant. The increase in PLR and NLR were caused by the increase in the platelet count, neutrophil count and the decrease in the lymphocyte count in peripheral blood just like in other systemic inflammatory reactive diseases. ${ }^{13-15}$ The result suggests that it is more useful to distinguish between $\mathrm{AE}$ and CE by monitoring the NLR value than by monitoring the total white blood cell count. This suggests that, although both parasites belong to the same genus and can damage the liver tissue of the host, the degrees of inflammation caused by them are different. This may be related to their completely different morphology and biological behavior. Echinococcus granulosus easily forms a fluid-filled hydatid cyst in the liver. The inner and outer surfaces of the capsule wall are called the germinal layer and cuticle, respectively. As time goes on, the eggs mature gradually, causing local inflammation. Finally, it is surrounded by fibrous tissues to form fluid-filled cystic lesions. The cyst structures of $\mathrm{AE}$ are similar to those of $\mathrm{CE}$, but the cysts are smaller, and can spread locally and distally to form tumor-like infiltrative lesions. ${ }^{16}$ The reasons for the significant increase in NLR may be that: 1 . In patients with hydatid infection, neutrophils promote inflammatory response and tissue damage by activating wide inflammatory mediators and inflammatory factors, that is, proinflammatory cytokines (interleukin-6 [IL-6], interleukin-8 [IL-8], $\alpha$-tumor necrosis factor $[\alpha$-TNF] $),{ }^{17}$ proteolytic enzymes (elastase, myeloperoxidase and collagenase) and reactive oxygen species (ROS). Neutrophils can also secrete vascular endothelial growth factor (VEGF), leading to angiogenesis and promoting the migration and invasion of Echinococcus multilocularis. 2. In turn, lymphocytes are a major component of the adaptive immune system, the decrease in peripheral lymphocyte count may be caused by apoptosis, necrosis and the redistribution of cells reflects the dysfunction of the immune system. Its reduction can be used as an independent marker of the progression of complications of inflammation, necrosis and sepsis. Accumulating data reveal that lymphopenia in patients with liver cancer and chronic liver diseases suggests poor prognosis. ${ }^{18}$ Immune-mediated liver injury is actually an important aspect of the pathogenesis of AE. Host infection with Echinococcus multilocularis has been proven to be a T lymphocyte-dependent process, ${ }^{12}$ after infection with Echinococcus multilocularis, the liver granuloma are formed, and a large number of lymphocytes are recruited around it, which results in a decrease in lymphocytes in peripheral blood. In most cases, peripheral CD4+ T lymphocytes are immature lymphocytes, many activated and differentiated CD4+ $\mathrm{T}$ lymphocytes gather in the inflammatory sites of the liver. ${ }^{19}$ The development of Echinococcus multilocularis is slow in the beginning stage, followed by a rapid increase in the size of the lesions in the middle/late stage, which is related to the overall reduction of lymphocyte function. Therefore, $\mathrm{T}$ lymphocyte failure is the mechanism by which pathogens survive without killing their hosts. A related study revealed that, ${ }^{20}$ the increase in NLR was related to the increase in a variety of interleukins, such as IL-6, interleukin-7 (IL-7), IL-8, interleukin-12 (IL-12), interleukin-17 (IL-17) and $\alpha$-TNF, which is an independent predictor of poor prognosis in patients with decompensated cirrhosis and hepatic cell carcinoma (HCC). They considered that the ability of NLR to predict tumor recurrence may be achieved through inflammatory tumor microenvironment.

PLR is similar to NLR. A study revealed that preoperative high PLR could predict the poor prognosis of HCC patients. $^{21}$ A previous study of the investigators confirmed that platelets not only played an important role in hemostasis and thrombosis but also helped regulate inflammation and immune response. More important is that platelets play a dual regulatory role in tissue inflammation. At first, platelets promote the occurrence of inflammation; they produce soluble substances to stimulate proliferation, differentiation and migration of Th1 and Th17 cells. In the process of eliminating inflammation, platelets inhibit the proliferation and differentiation of Th1 and Th17 cells by forming platelet-T lymphocyte aggregates. Platelet releasing factors, such as plateletderived growth factors, have been proven to enhance tumor progression and metastasis. AE is also called "insect cancer". Its metastasis is the same as a tumor. As a very important cytokine closely related to interleukin-23 (IL23), cytokines and other factors, IL-17 mediated proinflammatory immune response have been proven to promote the invasive growth in the tissues of parasites and their persistence in humans.

In addition, the levels of neutrophils, lymphocytes and platelets may be affected by some cytokines, such as IL-6 and IL-17. Besides determining the expression levels of inflammatory factors PLR and NLR, the correlation 
between the two and the clinicopathological parameters were also determined. PLR and NLR were positively correlated with ALT, AST, TIBL, DBIL, GGT and ALP respectively, their expressions were significantly higher in the $\mathrm{AE}$ group than in the other two groups, and were negatively correlated with ALB, and the ALB expression was significantly lower in the AE group than in the other two groups. The liver is the only place where ALB is produced. Serum ALB can reflect the synthesis function and total nutritional status of the liver. Acute and chronic inflammation can induce the decrease in ALB expression. The greater the inflammatory response, the lower the serum level of ALB is. With the deterioration of the liver function, ALB synthesis decreases. ALB is not only an indicator of acute and chronic inflammation but also an indicator of nutritional status. In the current study, the level of ALB was significantly lower in the AE and CE groups than in the control group and was lower in the $\mathrm{AE}$ group. The result suggests that there is severe malnutrition in AE patients. The result of this study revealed that ALT, AST, TIBL, DBIL, GGT and ALP levels lack the necessary sensitivity to predict the inflammatory response caused by Echinococcosis. The increase in these liver function indicators may only occur when the liver membrane permeability is deformed. This indicates severe histological damage rather than cirrhosis. Single point quantitative PLR measurement performed better in identifying AE patients (sensitivity: 81.8\%; specificity: $88.2 \%$ ).

This study still has the following limitations. Firstly, this study is a retrospective study, and not a randomized controlled trial. It is not blinded, therefore, there remains a certain risk of bias. Secondly, this study is a single-center clinical trial and the included sample size is small. Multi-center clinical trials with a larger sample size are still needed. Finally, this study did not evaluate the correlation between cytokines, inflammatory mediators with PLR and NLR; further confirmatory studies are needed to clarify the correlation.

\section{Conclusion}

This study first proves, based on clinical data, that PLR and NLR are increased significantly in patients with hydatid diseases, and they were significantly higher in AE patients than in CE patients. The type of hydatid can be determined by their values. PLR and NLR are independently correlated with the presence and severity of hydatid disease in our study population. The correlation of PLR is better than that of NLR. PLR can be calculated simply based on hemogram data, which is a simple, practical and cheap indicator. Therefore, PLR can be used as a useful marker to distinguish between $\mathrm{AE}$ and $\mathrm{CE}$ types and an increase in the inflammatory response and can also be used in the daily clinical practice of disease follow-up.

\section{Ethics Approval and Consent to Participate}

This study was conducted in accordance with the declaration of Helsinki. This study was conducted with approval from the Ethics Committee of Affiliated Hospital of Qinghai University. A written informed consent was obtained from all participants.

\section{Author Contributions}

All authors made substantial contributions to conception and design, acquisition of data, or analysis and interpretation of data; took part in drafting the article or revising it critically for important intellectual content; agreed to submit to the current journal; gave final approval of the version to be published; and agree to be accountable for all aspects of the work.

\section{Funding}

This work was supported by Qinghai Science and Technology Department Project (grant 2018-ZJ-757).

\section{Disclosure}

All authors have contributed significantly to the manuscript and declare that the work is original and has not been submitted or published elsewhere. None of the authors have any financial disclosure or conflicts of interest.

\section{References}

1. Wen H, Vuitton L, Tuxun T. Echinococcosis: advances in the $21 \mathrm{st}$ century. Clin Microbiol Rev. 2019;32(2):e00075-18. doi:10.1128/ CMR.00075-18

2. Abdulhameed MF, Habib I, Al-Azizz SA, Robertson I. A retrospective study of human cystic echinococcosis in Basrah province, Iraq. Acta Trop. 2018;178:130-133. doi:10.1016/j.actatropica.2017.11.011

3. Grubor NM, Jovanova-Nesic KD, Shoenfeld Y. Liver cystic echinococcosis and human host immune and autoimmune follow-up: a review. World J Hepatol. 2017;30:18-31.

4. Tamarozzi F, Mariconti M, Covini I, Brunetti E. Rapid diagnostic tests for the serodiagnosis of human cystic echinococcosis. Bull Soc Pathol Exot. 2017;110(1):20-30. doi:10.1007/s13149-017-0548-z

5. Jaiswal P, Jaiswal R, Attar BM, et al. Hepatobiliary and pancreatic: massive hepatic cystic echinococcosis compressing inferior vena cava. J Gastroenterol Hepatol. 2018;33(2):339. doi:10.1111/jgh.14038

6. Menteş A, Yalaz S, Killi R, Altintaş N. Radical treatment for hepatic echinococcosis. HPB. 2000;2(1):49-54. doi:10.1016/S1365-182X(17) 30701-3

7. Gerdes N, Zhu L, Ersoy M, et al. Platelets regulate CD4+ T-cell differentiation via multiple chemokines in humans. Thromb Haemost. 2011;106(2):353-362. doi:10.1160/TH11-01-0020 
8. Ying HQ, Deng QW, He BS, et al. The prognostic value of preoperative NLR, d-NLR, PLR and LMR for predicting clinical outcome in surgical colorectal cancer patients. Med Oncol. 2014;31(12):305 doi:10.1007/s12032-014-0305-0

9. Duman TT, Aktas G, Atak BM, Kocak MZ, Erkus E, Savli H. Neutrophil to lymphocyte ratio as an indicative of diabetic control level in type 2 diabetes mellitus. Afr Health Sci. 2019;19 (1):1602-1606. doi:10.4314/ahs.v19i1.35

10. Aktas G, Sit M, Dikbas O, et al. Elevated neutrophil-to-lymphocyte ratio in the diagnosis of Hashimoto's thyroiditis. Rev Assoc Med Bras. 2017;63(12):1065-1068. doi:10.1590/1806-9282.63.12.1065

11. Posul E, Yilmaz B, Aktas G, Kurt M. Does neutrophil-to-lymphocyte ratio predict active ulcerative colitis? Wien Klin Wochenschr. 2015;127(7-8):262-265. doi:10.1007/s00508-014-0683-5

12. Whaley CM, Pera MF, Cantor J, et al. Changes in health services use among commercially insured US populations during the COVID-19 pandemic. JAMA Netw Open. 2020;3(11):e2024984. doi:10.1001/ jamanetworkopen.2020.24984

13. Sit M, Aktas G, Erkol H, Yaman S, Keyif F, Savli H. Neutrophil to lymphocyte ratio is useful in differentiation of malign and benign thyroid nodules. P R Health Sci J. 2019;38(1):60-63. PMID: 30924917.

14. Bilgin S, Aktas G, Zahid kocak M, et al. Association between novel inflammatory markers derived from hemogram indices and metabolic parameters in type 2 diabetic men. Aging Male. 2019;1-5. doi:10.1080/13685538.2018.1434503

15. Seng JJB, Kwan YH, Low LL, Thumboo J, Fong WSW. Role of neutrophil to lymphocyte ratio (NLR), platelet to lymphocyte ratio (PLR) and mean platelet volume (MPV) in assessing disease control in Asian patients with axial spondyloarthritis. Biomarkers. 2018:1-12.
16. Mathur K, Kurbanova N, Qayyum R. Platelet-lymphocyte ratio (PLR) and all-cause mortality in general population: insights from national health and nutrition education survey. Platelets. 2019;1-6. doi:10.1080/09537104.2019.1549810

17. Díaz Á. Immunology of cystic echinococcosis (hydatid disease). $\mathrm{Br}$ Med Bull. 2017:1-13.

18. Freeman L, Guo H, David CN, Brickey WJ, Jha S, Ting JP. NLR members NLRC4 and NLRP3 mediate sterile inflammasome activation in microglia and astrocytes. $J$ Exp Med. 2017;214(5): jem.20150237. doi:10.1084/jem.20150237

19. Ding Y, Li P, He Q, et al. The CD4+T-lymphocyte count is an important predictor for the prognosis of cryptococcosis. Eur J Clin Microbiol Infect Dis. 2017;36(5):897-904. doi:10.1007/s10096-0162880-9

20. Bui JK, Halvas EK, Fyne E, et al. Ex vivo activation of CD4+ T-cells from donors on suppressive ART can lead to sustained production of infectious HIV-1 from a subset of infected cells. PLoS Pathog. 2017;13(2):e1006230. doi:10.1371/journal.ppat.1006230

21. Purcell S, Claus M, Hosgood G, Smart L. Interleukin-8, interleukin$1 \beta$ and tumour necrosis factor- $\alpha$ in sequential units of packed red blood cells collected from retired racing Greyhounds. Aust Vet J. 2017;95(1-2):26-32. doi:10.1111/avj.12549
Journal of Inflammation Research

\section{Publish your work in this journal}

The Journal of Inflammation Research is an international, peerreviewed open-access journal that welcomes laboratory and clinical findings on the molecular basis, cell biology and pharmacology of inflammation including original research, reviews, symposium reports, hypothesis formation and commentaries on: acute/chronic inflammation; mediators of inflammation; cellular processes; molecular mechanisms; pharmacology and novel anti-inflammatory drugs; clinical conditions involving inflammation. The manuscript management system is completely online and includes a very quick and fair peerreview system. Visit http://www.dovepress.com/testimonials.php to read real quotes from published authors. 